\section{Oral Abstracts}

\section{0-001 DWI-FLAIR MISMATCH DOES NOT PREDICT TIME OF STROKE ONSET IN DEFUSE 3 PATIENTS}

${ }^{1} \mathrm{D}$ Slawski*, ${ }^{2} \mathrm{~J}$ Heit, ${ }^{2} \mathrm{M}$ Marks, ${ }^{3} \mathrm{R}$ Lemmens, ${ }^{1} \mathrm{~L}$ Jakubowski, ${ }^{1} \mathrm{M}$ Mlynash, ${ }^{1} \mathrm{M}$ Lansberg, ${ }^{1} \mathrm{G}$ Albers. ${ }^{1}$ Neurology, Stanford Medicine, Palo Alto, CA; ${ }^{2}$ Radiology, Stanford Medicine, Palo Alto, CA; ${ }^{3}$ Neurology, KU Leuven, Leuven, BELGIUM

10.1136/neurintsurg-2020-SNIS.1

Introduction Acute stroke patients with unclear time of symptom onset have traditionally been ineligible for treatment with IV tissue plasminogen activator (tPA) based on a strict time window of treatment within 4.5 hours of symptom onset. Recently, the use of DWI-FLAIR mismatch to identify patients within the 4.5 hour time window has been suggested to broaden eligibility for tPA. Patients who are DWI positive and FLAIR negative qualify for treatment, while those who are both DWI and FLAIR positive are ineligible. In this study, we examined the MRI-selected patients in DEFUSE 3 and applied the rules for DWI-FLAIR mismatch ratings. DEFUSE 3 patients were all selected based on the presence of a PWI/ DWI mismatch. We hypothesized that 1) inter-rater agreement is suboptimal for the detection of DWI-FLAIR mismatch;2) the percentage of DWI-FLAIR mismatch patients is similar between known and unknown onset groups, and 3) intervention with mechanical thrombectomy (MT) yields favorable outcomes irrespective of DWI-FLAIR mismatch.

Methods This study examines the 30 patients from the DEFUSE 3 trial who were selected by PWI/DWI mismatch on MRI, rather than CT perfusion. Patients were either known to be $>6$ hours from symptom onset, had unknown time of symptom onset, or awoke with symptoms. Imaging was reviewed by two neuroradiologists and one vascular neurologist. Images were adjudicated in regard to whether a DWIFLAIR mismatch was present, blinded to all other clinical and imaging data. The percentage of reader agreement was assessed. The primary endpoint was functional independence $(0-2)$ on the 3 -month modified Rankin scale.

Results 30 patients were evaluated. Of these, 3 were excluded due to poor image quality. All patients (27/27) were found to have DWI positive lesions with 100\% agreement. All three readers agreed on FLAIR positivity in 9/27 patients $33 \%$ agreement). By consensus read, 15/27 patients were considered FLAIR positive (no DWI-FLAIR mismatch). Overall calculated inter-rater agreement was $72 \%$ for the detection DWI-FLAIR mismatch. In the unknown onset group, 8/14 (57\%) had no DWI-FLAIR mismatch by consensus read. In the known onset group, 7/13 (54\%) had no DWI-FLAIR mismatch. There were 7 patients with no DWI-FLAIR mismatch who underwent MT; 57\% achieved functional independence, which compares favorably with the overall study rate of $45 \%$ in the MT group. In contrast, patients with no DWI-FLAIR mismatch who did not receive MT had a favorable outcome rate of $12.5 \%(1 / 8)$.

Conclusions In this study, the inter-rater agreement for detection of FLAIR-DWI mismatch among skilled readers was suboptimal. The rate of DWI-FLAIR mismatch did not differ in the known $>6$ hrs vs unknown onset patients. Nearly half of the patients in the known $>6$ hrs group did not have DWIFLAIR mismatch, suggesting that FLAIR positivity is not a sensitive marker for being $>6$ hours from onset in patients with PWI/DWI mismatch. The absence of a DWI-FLAIR mismatch does not preclude a favorable DWI/PWI mismatch or favorable outcomes following late-window MT.

Disclosures D. Slawski: None. J. Heit: None. M. Marks: None. R. Lemmens: None. L. Jakubowski: None. M. Mlynash: None. M. Lansberg: None. G. Albers: None.

\section{0-002 FAVORABLE VENOUS MICROPERFUSION PROFILE CORRELATES WITH PIAL ARTERIAL COLLATERAL STATUS AND CLINICAL OUTCOME IN ACUTE STROKE PATIENTS WITH LARGE VESSEL OCCLUSION}

${ }^{1} \mathrm{~T}$ Faizy ${ }^{*},{ }^{1} \mathrm{R}$ Kabiri, ${ }^{1} \mathrm{M}$ Leipzig, ${ }^{1} \mathrm{~S}$ Christensen, ${ }^{2} \mathrm{G}$ Broocks, ${ }^{2} \mathrm{~F}$ Flottmann, ${ }^{3} \mathrm{M}$ Lansberg, ${ }^{3} \mathrm{G}$ Albers, ${ }^{2} \mathrm{~J}$ Fiehler, ${ }^{1} \mathrm{M}$ Wintermark, ${ }^{1} \mathrm{~J}$ Heit. ${ }^{1}$ Neuroimaging and Neurointervention, Stanford University, Stanford, $C A_{;}{ }^{2}$ Neuroimaging and Neurointervention, University Medical Center Hamburg-Eppendorf, Hamburg, GERMANY; ${ }^{3}$ Neurology, Stanford University, Stanford, $C A$

\subsection{6/neurintsurg-2020-SNIS.2}

Purpose Robust pial arterial collaterals (PAC) preserve blood flow to critically hypoperfused brain tissue in patients with acute ischemic stroke due to large vessel occlusion (AIS-LVO). CT angiography (CTA) based methods of pial collateral assessment do not provide tissue level perfusion information, and prior studies have shown that PAC assessment on CT perfusion imaging strongly predicts outcome in AIS-LVO patients treated by thrombectomy. Patients with favorable pial collaterals and brain tissue perfusion also likely have robust cortical venous drainage relative to patients with more impaired cerebral perfusion. We determined the venous microperfusion profile (VMP) in AIS-LVO patients. We hypothesized that robust PAC on CT perfusion predict robust cortical venous contrast opacification on pre-treatment CTA and that a favorable VMP is associated with good clinical outcomes in AIS-LVO patients. Materials and Methods We performed a multicenter retrospective cohort study of consecutive AIS-LVO patients who underwent thrombectomy. Included patients had interpretable prethrombectomy CT angiography (CTA) and CT perfusion (CTP) studies and clinical outcome data. Patient details were obtained from prospectively maintained stroke databases and the electronic medical record. Pre-thrombectomy CTA and CTP studies were reviewed and scored for tissue-level collaterals using the Hypoperfusion Intensity Ratio (HIR). HIR was defined as the volume ratio of brain tissue with [Tmax $>10$ sec/ Tmax $>6 \mathrm{sec}]$ such that a lower HIR correlates with favorable collaterals. HIR was automatically calculated by RAPID (iSchemaView). VMP was determined by opacification of the vein of Labbé, sphenoparietal sinus, and superficial middle cerebral vein on CTA as: 0 , not visible; 1 , moderate opacification; and 2, full. Primary outcome measure was VMP. Secondary outcome measure was ordinal modified Rankin Scale (mRS). Ordinal linear regression models were performed to predict the effect of HIR on VMP, as well as the effect of VMP on mRS.

Results 186 patients met inclusion criteria. HIR was dichotomized into lower $(\leq 0.4$, good collaterals) and higher $(\geq 0.5$, poor collaterals) ratios. Mann-Whitney-U test indicated that subjects with higher HIR (median COVES = 1) had lower VMP than patients with lower HIR (median COVES = 3) $(p<0.001)$. In an ordinal logistic regression model, we tested the effects of VMP on mRS at 90 days after discharge while controlling for HIR (nondichotomized), age, and TICI score. High (favorable) VMP predicted lower (favorable) mRS 
$(\mathrm{OR}=0.544,[95 \%$ CI $0.4-0.7] ; \mathrm{p}=0.032)$, which indicates that patients with robust VMP had better neurological outcomes 90 days after discharge.

Conclusion A robust cerebral venous microperfusion profile reflects greater tissue microperfusion, good arterial collateralization status and is associated with improved clinical outcome in patients with AIS.

Disclosures T. Faizy: None. R. Kabiri: None. M. Leipzig: None. S. Christensen: None. G. Broocks: None. F. Flottmann: None. M. Lansberg: None. G. Albers: None. J. Fiehler: None. M. Wintermark: None. J. Heit: None.

\section{0-003 RESULTS OF THE STROKE THROMBOEMBOLISM REGISTRY OF IMAGING AND PATHOLOGY: A MULTICENTER INTERNATIONAL STUDY}

${ }^{1} \mathrm{~W}$ Brinjikji ${ }^{*},{ }^{2} \mathrm{R}$ Nogueira, ${ }^{3} \mathrm{R}$ Hanel, ${ }^{4} \mathrm{~K}$ Layton, ${ }^{5}$ J Delgado, ${ }^{6} \mathrm{M}$ Gounis, ${ }^{7} \mathrm{~A}$ Yoo, ${ }^{8} \mathrm{~V}$ Pereira, ${ }^{9} \mathrm{M}$ Almekhlafi, ${ }^{10} \mathrm{~K}$ Doyle, ${ }^{11} \mathrm{~B}$ Patel, ${ }^{12} \mathrm{~B}$ Jahromi, ${ }^{13} \mathrm{P}$ Kvamme, ${ }^{1} \mathrm{R}$ Kadivel, ${ }^{1} \mathrm{D}$ Dai, 'D Kallmes, 'S Fitzgerald. 'Radiology, Mayo Clinic, Rochester, MN; ${ }^{2}$ Neurology, Grady Memorial Hospital, Atlanta, GA; ${ }^{3}$ Neurosurgery, Baptist, Jacksonville, FL; ${ }^{4}$ Radiology, Baylor Scott White Hospital, Dallas, TX; ${ }^{5}$ Radiology, Abbott Northwestern Hospital, Minneapolis, $M N ;{ }^{6}$ Radiology, University of Massachussets, Worcestire, MA; ${ }^{7}$ Radiology, UT Houston, Houston, $T X_{;}{ }^{8}$ Neurosurgery, University of Toronto, Toronto, ON, CANADA; ${ }^{9}$ Neurology, University of Calgary, Calgary, AB, CANADA; ${ }^{10}$ Physiology, University of Galway, Galway, IRELAND; ${ }^{11}$ Radiology, Carillion Clinic, Carillion, WV; ${ }^{12}$ Neurosurgery, Northwestern Memorial Hospital, Chicago, IL; ${ }^{13}$ Radiology, UT Knoxville, Knoxville, TN

\subsection{6/neurintsurg-2020-SNIS.3}

Background We performed a multicenter prospective clinical registry across 12 centers to study the association between histopathological characteristics of retrieved clots and imaging, stroke etiology and clinical outcomes.

Materials and Methods Following IRB approval at the 12 centers, patients were enrolled in the STRIP registry. All retrieved emboli were sent for histopathological analysis with H\&E and MSB staining. Demographic variables, comorbidities, stroke etiology, imaging findings and procedural details were collected for each case. We studied the association between clot histopathology and imaging findings, stroke etiology and and revascularization outcomes. Student's t-test was used for continuous variables and chi-squared testing for categorical variables.

Results To date, 1457 patients have been included. Platelet rich clots were associated with a significantly lower rate of first pass TICI $2 \mathrm{c} / 3$ revascularization than platelet poor clots $(\mathrm{OR}=0.68,95 \% \mathrm{CI}=0.48-0.88, \mathrm{P}<0.0001)$. The rate of first pass TICI $2 \mathrm{c} / 3$ recanalization was $41 \%$ with aspiration compared to $26 \%$ for stent-triever and $15 \%$ for combined techniques with platelet rich clots $(\mathrm{P}<0.0001)$. There was a significant correlation between platelet rich clots and the absence of hyperdensity on non-contrast CT $(p=0.003)$ and a significant inverse correlation between the percentage of platelets and mean HU on NCCT $(r=-0.243, p=0.005)$. We found that a Hounsfield unit cutoff of 55 or less had a sensitivity and specificity of $70 \%$ for a platelet rich clot. We did not notice any interaction between clot composition and stroke etiology.

Conclusions Interim analysis of the STRIP registry suggests that the platelet content of a clot may be the most revealing factor in determining a clot's imaging features and revascularization outcome. Platelet rich clots are less dense on NCCT and are associated with poorer first pass TICI 2c/3. Aspiration therapy was associated with higher rates of first pass recanalization than stent-retriever thrombectomy or combined techniques.

Disclosures W. Brinjikji: None. R. Nogueira: None. R. Hanel: None. K. Layton: None. J. Delgado: None. M. Gounis: None. A. Yoo: None. V. Pereira: None. M. Almekhlafi: None. K. Doyle: None. B. Patel: None. B. Jahromi: None. P. Kvamme: None. R. Kadirvel: None. D. Dai: None. D. Kallmes: None. S. Fitzgerald: None.

\section{0-004 PROSPECTIVE, MULTI-CENTERED, EMS-ADMINISTERED, PRE-HOSPITAL VALIDATION STUDY OF THE RAPID ARTERIAL OCCLUSION EVALUATION (RACE) SCALE FOR DETECTING LARGE VESSEL OCCLUSION STROKE IN THE UNITED STATES COMPARED TO THE ORIGINAL RACE VALIDATION STUDY FROM SPAIN: A SUBANALYSIS OF THE PREDICT STUDY}

${ }^{1} \mathrm{~A}$ Cruz*, ${ }^{1} \mathrm{E}$ Fortuny, ${ }^{1} \mathrm{~B}$ Ugiliweneza, ${ }^{1} \mathrm{D}$ Wang, ${ }^{2} \mathrm{~A}$ White, ${ }^{1} \mathrm{~N}$ Khattar, ${ }^{1} \mathrm{~S}$ Adams, ${ }^{1} \mathrm{~B}$ Gallinore, ${ }^{1} \mathrm{D}$ Ding, ${ }^{3} \mathrm{~S}$ Wolfe, ${ }^{4} \mathrm{D}$ Heck, ${ }^{1} \mathrm{R}$ James. ${ }^{1}$ Neurosurgery, University of Louisville, Louisville, KY; ${ }^{2}$ Radiology, University of Louisville, Louisville, KY; ${ }^{3}$ Neurosurgery, Wake Forest Baptist Health, Lexington, NC; ${ }^{4}$ Radiology, Forsythe Medical Center, Winston-Salem, NC

\subsection{6/neurintsurg-2020-SNIS.4}

Introduction Pre-hospital identification of patients with large vessel occlusion (LVO) is critical in the timely triage of thrombectomy-eligible LVO patients to comprehensive stroke centers. The Rapid Arterial oCclusion Evaluation (RACE) scale was prospectively validated to identify LVO patients by EMS in Spain and has been widely adopted in the United States, though these healthcare infrastructure and EMS systems may differ significantly and these results may not be completely

Abstract 0-004 Table 1 Sensitivity, Specificity, Accuracy, PPV and NPV values for the RACE scale across various cut-off values for PREDICT (U. $S$ based study) and the Original RACE study (Spain Based) PPV=Positive Predictive Value, Negative Predictive Value

\begin{tabular}{|c|c|c|c|c|c|c|c|c|c|c|}
\hline & \multicolumn{9}{|l|}{ RACE Scale (U.S Based study) } & \multicolumn{4}{|l|}{ RACE scale (Original Spanish Based Study) } \\
\hline $\begin{array}{l}\text { RACE } \\
\text { Score }\end{array}$ & Sensitivity & Specificity & PPV & NPV & Accuracy & Sensitivity & Specificity & PPV & NPV & Accuracy \\
\hline$\geq 1$ & 1.00 & 0.149 & 0.119 & 1.00 & 0.237 & 1.00 & 0.13 & 0.24 & 1.00 & 0.31 \\
\hline$\geq 2$ & 0.958 & 0.303 & 0.137 & 0.984 & 0.371 & 0.97 & 0.27 & 0.27 & 0.97 & 0.42 \\
\hline$\geq 3$ & 0.875 & 0.519 & 0.174 & 0.973 & 0.556 & 0.93 & 0.40 & 0.30 & 0.96 & 0.51 \\
\hline$\geq 4$ & 0.833 & 0.625 & 0.204 & 0.970 & 0.647 & 0.89 & 0.55 & 0.35 & 0.95 & 0.62 \\
\hline$\geq 5$ & 0.792 & 0.716 & 0.244 & 0.968 & 0.724 & 0.85 & 0.68 & 0.42 & 0.94 & 0.72 \\
\hline$\geq 6$ & 0.750 & 0.784 & 0.286 & 0.964 & 0.780 & 0.72 & 0.77 & 0.46 & 0.91 & 0.76 \\
\hline$\geq 7$ & 0.500 & 0.865 & 0.300 & 0.938 & 0.828 & 0.53 & 0.89 & 0.56 & 0.87 & 0.81 \\
\hline$\geq 8$ & 0.250 & 0.947 & 0.353 & 0.916 & 0.875 & 0.32 & 0.95 & 0.65 & 0.84 & 0.82 \\
\hline $\mathbf{9}$ & 0.167 & 0.986 & 0.571 & 0.911 & 0.901 & 0.07 & 0.99 & 0.56 & 0.79 & 0.79 \\
\hline
\end{tabular}

